20. Breuckmann F, Hochadel M, Darius H et al (2015) Guideline-adherence and perspectives in the acute management of unstable angina - Initial results from the German chest pain unit registry. J Cardiol 66(2):108-113

21. Breuckmann F, Hochadel M, Münzel T et al (2015) Timing of percutaneous coronary intervention in troponin-negative patients with acute coronary syndrome without persistent ST-segment elevation: preliminary results and status quo in German chest pain units. Crit Pathw Cardiol 14(1):7-11

22. Pollack CV Jr, Hollander JE, Chen AY et al (2009) Non-ST-elevation myocardial infarction patients who present during off hours have higher risk profiles and are treated less aggressively, but their outcomes are not worse: a report from Can Rapid Risk Stratification of Unstable Angina Patients Suppress ADverse Outcomes with Early Implementation of the ACC/AHA Guidelines CRUSADE initiative. Crit Pathw Cardiol 8(1):29-33

23. Illmann A, Riemer T, Erbel R et al (2014) Disease distribution and outcome in troponin-positive patients with or without revascularization in a chest pain unit: results of the German CPURegistry. Clin Res Cardiol 103(1):29-40

24. Maier LS, Darius H, Giannitsis E et al (2013) The German CPU Registry: comparison of troponin positive to troponin negative patients. Int J Cardiol 168(2):1651-1653

25. Borna C, Thelin J, Ohlin B (2014) High-sensitivity troponin $\mathrm{T}$ as a diagnostic tool for acute coronary syndrome in the real world: an observational study. Eur J Emerg Med 21(3):181-188

26. Thiele H, Rach J, Klein N et al (2012) Optimal timing of invasive angiography in stable nonST-elevation myocardial infarction: the Leipzig Immediate versus early and late PercutaneouS coronary Intervention triAl in NSTEMI (LIPSIANSTEMI Trial). Eur Heart J 33(16):2035-2043

\title{
Erratum
}

Herz 2016 $41: 731$

DOI 10.1007/s00059-016-4507-4

Online publiziert: 7. November 2016

๑) Springer Medizin Verlag Berlin 2016

CrossMark

\author{
A. Schmermund · J. Eckert · S. N. Schelle · H. Eggebrecht
}

Cardioangiologisches Centrum Bethanien, Frankfurt am Main, Deutschland

\section{Erratum zu: Bildgebung bei struktureller Herzerkrankung}

\section{Bedeutung für die interventionelle Therapie}

\section{Erratum zu: \\ Herz (2016) 41:639-652 \\ DOI 10.1007/s00059-016-4481-x}

In $\bullet$ Abb. 1 ist leider ein Fehler vorhanden. Die Orientierung der P1/A1- und $\mathrm{P} 3 / \mathrm{A} 3$-Segmente ist verkehrt herum dargestellt.

Wir bitten den Fehler zu entschuldigen.

\section{Korrespondenzadresse}

\section{Prof. Dr. A. Schmermund}

Cardioangiologisches Centrum Bethanien Im Prüfling 23, 60389 Frankfurt am Main, Deutschland a.schmermund@ccb.de

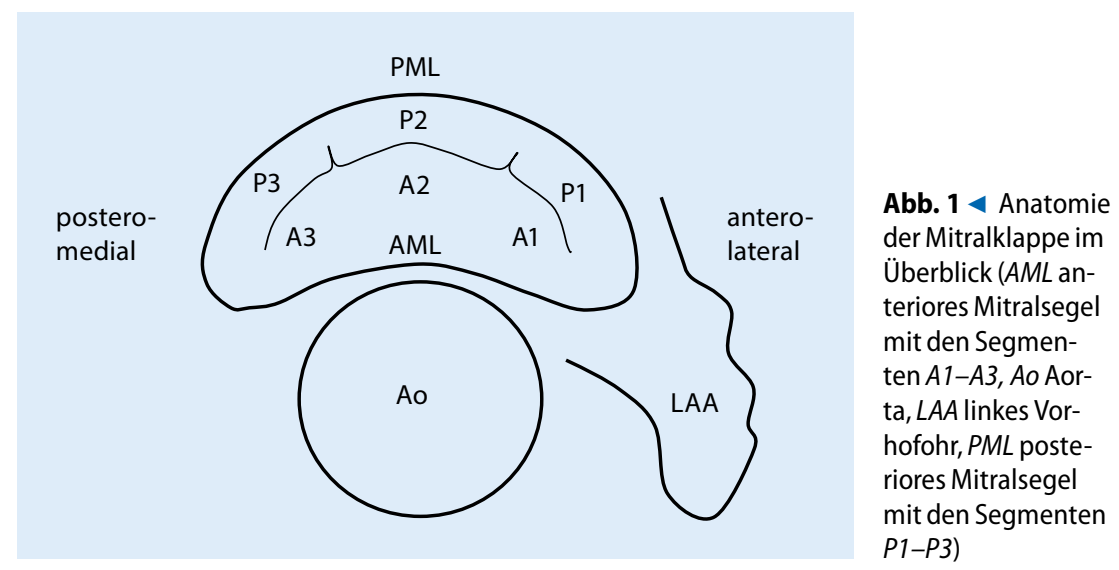

Die Online-Version des Originalbeitrages finden Sie unter http://dx.doi.org/10.1007/s00059016-4481-x 